\title{
Hippocampal Maturation Drives Memory from Generalization to Specificity
}

\author{
Attila Keresztes, ${ }^{1, \star}$ Chi T. Ngo, ${ }^{2}$ Ulman Lindenberger, ${ }^{1,3}$ Markus Werkle-Bergner, ${ }^{1}$ and \\ Nora S. Newcombe ${ }^{2}$
}

During early ontogeny, the rapid and cumulative acquisition of world knowledge contrasts with slower improvements in the ability to lay down detailed and long-lasting episodic memories. This emphasis on generalization at the expense of specificity persists well into middle childhood and possibly into adolescence. During this period, recognizing regularities, forming stable representations of recurring episodes, predicting the structure of future events, and building up semantic knowledge may be prioritized over remembering specific episodes. We highlight recent behavioral and neuroimaging evidence suggesting that maturational differences among subfields within the hippocampus contribute to the developmental lead-lag relation between generalization and specificity, and lay out future research directions.

The Hippocampus at Center Stage: Generalization and Specificity

The fact that infants and young children are prodigious learners creates one of the most perplexing paradoxes of human ontogeny: in the first 2 years of life, babies acquire much world knowledge and show rapid vocabulary acquisition [1]. However, they are not across-the-board learners. Despite substantial gains in semantic memory (see Glossary), episodic memory in infants is characterized by dense amnesia for events in their lives. To a lesser extent, this paradox continues through preschool and early elementary school, and even into middle childhood. The slow and protracted development of spatial and temporal resolution of memories [1-4] occurs in parallel with continued increases in world knowledge. These observations highlight a primary conflict in the goals of all adaptive learning systems: detecting regularities in the world through generalization versus encoding and remembering particular events and their details through mnemonic specificity.

This distinction can be captured by functionalist theories that conceptualize memory as a set of processes that are not independent from processes of other cognitive systems $[5,6]$, as well as by classical taxonomies of memory systems such as the distinction between episodic and semantic memory $[7,8]$. These theories are not fully overlapping. For instance, semantic knowledge about a particular topic can be richly detailed, hence specific, but the person possessing this knowledge may not have access to the individual episodes from which this knowledge has been extracted. In addition, autobiographical memories can be relatively general. For instance, one might remember last year's birthday party without being able to recall the type of cake served, not to mention its taste [9]. We argue that the functionalist distinction between memory specificity and generalization better captures such instances than the semantic-episodic distinction, and, more importantly, that understanding the development of cognitive and neural processes supporting specificity and generalization may provide a mechanistic explanation for the apparent discrepancy between rapid knowledge acquisition and the slow development of memory for details that is observed during childhood.
Highlights

High-resolution MRI allows in vivo investigation of regions within the hippocampus in humans.

Human hippocampal maturation continues beyond middle childhood and potentially into adolescence. It is characterized by an uneven maturational pace of different structures within the hippocampus.

Generalization and specificity are useful concepts pointing to component processes of memory that allow the integrated study of learning and memory during childhood.

Novel behavioral techniques provide estimates of the integrity of neural computations supporting generalization and specificity.

${ }^{1}$ Center for Lifespan Psychology, Max Planck Institute for Human Development, Berlin, Germany ${ }^{2}$ Department of Psychology, Temple University, Philadelphia, PA, USA ${ }^{3}$ Max Planck-University College London (UCL) Centre for Computational Psychiatry and Ageing Research, London, England, and Berlin, Germany

${ }^{*}$ Correspondence:

keresztes@mpib-berlin.mpg.de (A. Keresztes). 


\section{Box 1. Developmental Cognitive Neuroscience of Memory}

Historically, the focus in research on memory development during middle childhood and adolescence had been on extrahippocampal brain areas. Initial views on memory suggested major dissociations between forms - episodic versus semantic [73]) - or systems - declarative versus nondeclarative [74] - of memory. These divisions were partly rooted in presumed differential reliance on the hippocampus $[7,8,75]$. Today, it is generally recognized that distinctions based on the presence or lack of hippocampal involvement may be insufficient to fully characterize mnemonic phenomena. This insight was fostered by research that departed from the localizationist approach to addressing brain systems and related cognitive content, and instead adopted a component process approach addressing computational mechanisms [13].The centrality of hippocampal computations has now been established in both episodic and semantic memories [76,77], in working memory (e.g., [78,79]), and in a broad range of non-mnemonic functions [15].

Despite these recent advances, the distinction between hippocampus-dependent episodic memory and non-hippocampus-dependent semantic memory continues to inform developmental memory research (e.g., [80]). At the same time, it has been recognized that episodic memories are not only dependent on the hippocampus but also on a network of brain areas, and most prominently on the prefrontal cortex [81]. In line with available data indicating that the hippocampus matures relatively early compared to the prefrontal cortices $[17,19,82]$, research on the neural underpinnings of memory development during childhood has focused on the contribution of extrahippocampal areas to developmental gains in recollection [45], in source memory [46], or in schema-supported memory [72,83]. Given that generalization-like phenomena (e.g., familiarity, gist-based memories) are, compared to recollection, relatively stable across middle childhood [84-86], the role of hippocampal maturation in the development of generalization has rarely been studied. This stance was consistent with computational theories of the time suggesting that generalization depends on the cortex, and not the hippocampus [10]. In sum, the role of hippocampal maturation in memory development beyond early childhood has not been in the focus of research on memory development - until recent discoveries of the protracted development of the hippocampus (e.g., [38,52]).

Our goal here is to show that a functionalist approach to memory development provides new insights into the orderly progression of memory development during childhood. Our approach is based on computational models of generalization and specificity [10-12], on recent discoveries concerning hippocampal development, and is partly inspired by a component process framework to memory [13], which envisages memory as an interaction of processes tied to functionally and structurally distinct components. With this paper, we would like to put the hippocampus - its ontogeny, structure, and function - at center stage (Box 1).

\section{Computational Models of Generalization and Specificity}

From the first day of life, the human brain keeps a balance between generalization and specificity. Generalization supports the extraction of regularities across repeated exposures to similar experiences, and specificity allows the formation and retrieval of particular experiences with rich detail, distinct from the backdrop of overlapping memories [10-12].

Classically, complementary learning systems theory (CLS [10]) has offered a computational framework for understanding the interplay of generalization and specificity. CLS postulates that generalization is achieved through slow neocortical learning, whereas hippocampal computations are necessary for achieving specificity. CLS has proved to be highly influential in memory research and offers a mechanistic explanation for a wide range of memory phenomena. However, there are a few observations and empirical data that it cannot accommodate. First, in some cases generalization occurs rapidly and either involves the hippocampus, or even depends on it [14-16]. Second, several cortical areas relevant for memory, in particular the prefrontal cortex, mature slowly during childhood and adolescence [17-19]. CLS alone fails to explain how young children are able to put a premium on generalization at the expense of specificity, given that the brain areas assumed to support rapid generalization are particularly slow to mature.

More recent computational models explicitly model hippocampal involvement to resolve the fast generalization problem. An update of the CLS theory, the REMERGE model ([11], see also

\section{Glossary}

Complementary learning systems theory (CLS): a theoretical framework of memory based on computational modeling $[10,20]$. Its original formulation posited that through pattern separation, the hippocampus allows fast encoding of specific representations, and that the neocortex, through its distributed nature, allows slow extraction of generalized knowledge. In this framework, the hippocampus and cortex act in a complementary fashion to implement specificity and generalization.

Episodic memory: a form of human memory that refers to memory for spatially and temporally anchored events.

Generalization: we use the term generalization at two levels. At the computational level, we refer to it to describe processes that enable the brain to extract an underlying structure from repeated exposures to similar input patterns, and use the term to refer specifically to hippocampal generalization. At the cognitive level, we refer to the ability to extract regularities across repeated events.

\section{Mnemonic similarity task: a} recognition memory task to assess the integrity of neural pattern separation. There are two common task variants - an incidental encoding version, where encoding is followed by an 'old'/'similar'/'new' memory judgment test ([32]; discussed in this article) (Figure 1G, $\mathrm{H})$, and a continuous recognition version [97].

Relational memory: memory for at least two items (features, objects, concepts, locations, etc.) that are related to one another on the basis of conceptual, spatial, or temporal associations. The term originated in animal research and is widely used in cognitive neuroscience. The term associative memory emerged in cognitive psychology, and is primarily used interchangeably with the term relational memory. In cognitive neuroscience, associative memory is often defined as memory for cooccurrences, and is differentiated from other types of relational memory for locations and temporal sequences. 
[20]), proposes that generalization is supported by recurrent processing within the total hippocampal network. Within such a 'big loop', specific input patterns in the hippocampus can interact with cortical representations, allowing fast generalization to take place. A different modification to CLS described in [12] instantiates generalization and specificity along the monoand trisynaptic pathways within the hippocampal circuit. In line with emerging computational models of generalization within the hippocampus, neuroimaging and behavioral data suggest that construction of generalized memories is supported by the integration of current and previous experiences in concert with hippocampally supported encoding processes [21,22].

Major differences notwithstanding, most current computational models of hippocampal function have inherited a core principle from Marr's early mathematical memory model [23]: the computations of pattern separation and pattern completion. In current models, supported by abundant data from animal and human studies (reviewed in [24-26]), pattern separation allows the hippocampus to dissociate input from previously stored, but highly similar, representations, whereas pattern completion allows partial input to be integrated into previously stored representations. We acknowledge that pattern separation and completion can be linked to specificity and generalization in more than one way (Box 2). We subscribe to the view that pattern separation is necessary for specificity, whereas pattern completion supports generalization. Importantly, intrahippocampal subfields are differentially involved in these processes. Specifically, the dentate gyrus (DG) has been linked to pattern separation, whereas among the cornu ammoni (CA) regions, CA3 has been linked to both pattern separation and pattern completion, and CA1 has been linked to pattern completion [25].

These insights into complementary computational properties of different subfields of the hippocampus have important implications for understanding memory development in childhood and adolescence. They open the possibility that the developmental precedence of generalization over specificity is brought about by differences in maturational pace among

\section{Box 2. Pattern Separation and Pattern Completion}

In perhaps the most influential recent review on pattern separation and completion in the hippocampus [25], based on [23], the authors define pattern completion as the 'process by which incomplete or degraded representations are filledin based on previously stored representations', thus allowing generalization. Pattern separation in turn is defined as a process 'whereby similar representations are stored in a distinct, non-overlapping (orthogonalized) fashion'. Importantly, the definitions vary in the literature. Some adopt a view that the product of pattern completion is a previously stored representation rebuilt from available input serving as partial cues [24,87-93]. Others define pattern completion more broadly and suggest that stored representations can also support the extraction of a common representation that enables generalizations across experiences or binding during encoding $[16,32,37,38,94]$. Note the often implicit conception (or misconception [24]) in these definitions, according to which pattern separation and completion are not separate processes but instead comprise two ends of a continuum. By contrast, it has been suggested that the converse of pattern separation is pattern convergence - that is, cases when the correlation between two patterns of cell population activity evoked by two different input stimuli in a given region increases in a downstream region [95].

A major source of inconsistencies is that pattern separation and completion are studied at different levels of analysis spanning animal and computational models, human neuroimaging, and experimental psychology. Comparisons of concepts across levels of analysis will necessarily fall through unless inter-level relations of concepts are addressed. For instance, some researchers advocate the view that pattern separation is a storage process whereas pattern completion is a retrieval process [24]. However, such a distinction cannot accommodate the observation of cognitive psychology that memories are constructive in nature [96]. In this view, pattern completion is necessary to construct novel memories based on generalizations. In addition, computationally, pattern separation and completion have been ascribed to distinct hippocampal structures. However, non-invasive neuroimaging and behavioral studies can only collect indirect evidence for the two processes and are confounded in currently available measures [32-34]. For instance, although evidence supports the assumption that discrimination performance on the mnemonic similarity task reflects the integrity of neural pattern separation $[33,35,36]$, pattern completion necessarily contributes to performance with an unknown weight $[32,38]$. Future research needs to confirm whether these processes can be disentangled from one another at neural and cognitive levels of analysis, and to better link different levels of analysis.
REMERGE model: REMERGE stands for 'recurrency and episodic memory results in generalization' $[11,20]$. It is a modification of CLS that accommodates findings suggesting that, in addition to the neocortex, the hippocampus is also involved in generalization. This is achieved by adding recurrent connections within the hippocampus. Semantic memory: a form of human memory that refers to factual knowledge about the world.

Source memory: the spatial, temporal, and other types of anchors for an episodic memory. For instance, the exact location, the time of day of hearing a story, and the person telling us the story are all source memories for the story. Broadly, this is a type of associative/ relational memory.

Specificity: we define specificity at two levels. At the computational level, we refer to it to describe processes that enable the brain to store and reinstate highly specific activity patterns in the face of overlapping elements with other patterns, and use the term to refer only to hippocampal specificity. At the cognitive level, we refer to the ability to encode and retrieve memories with highly specific details. 
regions within the hippocampus. To substantiate this claim, we provide evidence that pattern completion precedes pattern separation during childhood development, and report initial evidence suggesting that this developmental lag is indeed associated with differential development of regions within the hippocampus.

\section{Changing Balance between Pattern Completion and Pattern Separation}

The imbalance between assimilation and accommodation during early and middle childhood, in which children tend to extract schematic knowledge at the expense of learning and recollecting specific events, was actually noted many years ago by Piaget $[27,28]$. This imbalance has resurfaced in computational models of memory [10], and later as the imbalance between pattern completion and pattern separation $[11,25,29]$. Studies on the development of pattern separation and completion in childhood are scarce, but their results converge. These studies have adapted experimental paradigms originally developed for rodents $[30,31]$ and human adults $[32,33]$ to assess pattern separation and completion in children. One of the first studies on pattern separation development found that the ability of children aged 1.5-4 years to discriminate between close locations in a complex spatial memory task improved with age [34] (Figure 1A-F). These improvements in the spatial resolution of memory suggested a first link to the development of pattern separation abilities.

Two recent studies from our laboratories assessed pattern separation development with adaptations of the mnemonic similarity task [33] (Figure 1G,H). This task assesses mnemonic discrimination, the ability to distinguish between highly similar memories, and has been shown to be a reliable index of pattern separation integrity $[33,35,36]$. A first study [37] investigated mnemonic discrimination and relational memory (assessed by a task requiring binding of items, to items and to contexts) in children aged 4 and 6 years, as well as in young adults. Importantly, relational memory performance and mnemonic discrimination abilities were uncorrelated. Moreover, although improvements on the relational memory task were only noted until the age of 6 , mnemonic discrimination improved from 4 to 6 years of age and beyond. In a second study [38], children aged $6-14$ years and young adults performed a mnemonic similarity task, as well as tasks assessing memory for items, item-item associations, and source memory. The results revealed a heterogeneous pattern of memory development across the full sample, with most measures, including mnemonic discrimination, showing improvements across childhood. In line with [37], mnemonic discrimination did not correlate with memory for inter-item associations. In addition, relational memory (as indexed by source memory performance) and pattern separation were dissociated in terms of their structural brain correlates across the developmental sample: source memory development was specifically related to maturation of frontal cortex, whereas mnemonic discrimination development was specifically related to maturation of the hippocampus.

As noted above (also Box 2), we subscribe to the view that mnemonic discrimination relies on pattern separation supporting memory specificity, whereas relational memory relies on pattern completion and additional cortical areas supporting generalization. Thus, taken together, available behavioral age trends and their associations to structural brain differences support the notion that pattern separation develops on different trajectories from pattern completion. This is not to imply that only pattern separation develops in this period, whereas pattern completion does not. For instance, a recent study [39] provided evidence that performance on tasks relying on generalization through integrating separate experiences, such as associative inference and statistical learning, does develop from 6 to 30 years of age. However, based on the available evidence, we posit a shift in the balance between pattern completion and pattern separation towards the latter. We next summarize evidence suggesting that the shift in the 


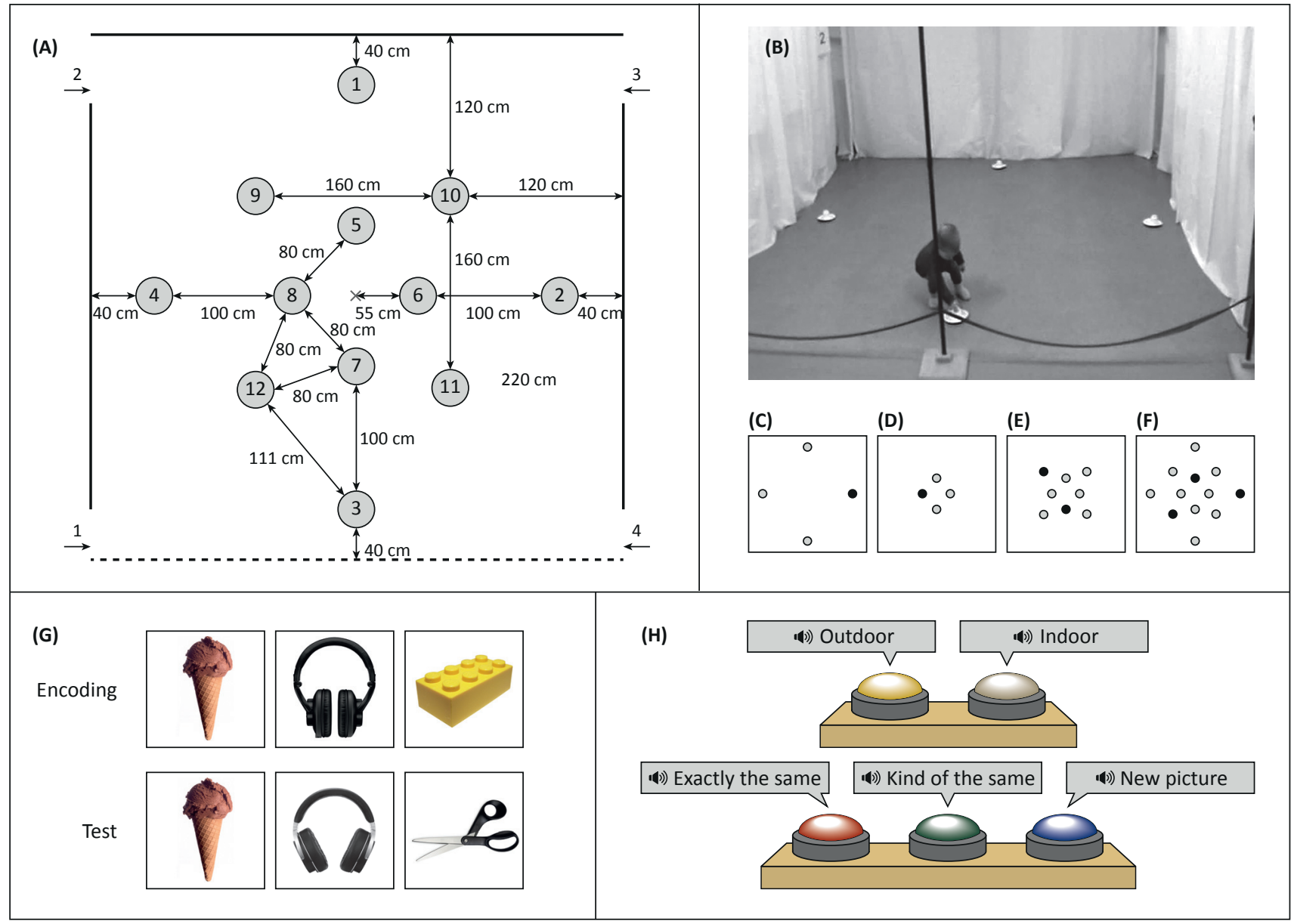

Trends in Cognitive Sciences

Figure 1. Tasks Assessing Pattern Separation Development. (A-F) Task used to show age-related differences in spatial pattern separation development in children aged 18 to 48 months in ([34], adapted from [33]). (A) Children had to memorize spatial locations that had been associated with a reward. (B) They then were tested in different versions of the same environment, with combinations of rewarded (filled circles) and non-rewarded locations being present in the environment in increasing complexity (C-F). Thus, this study manipulated the number of rewarded locations and the spatial resolution necessary to separate rewarded from nonrewarded cues: for example, (C) and (D) show environments with one rewarded location, but (D) requiring a higher spatial resolution. (G,H) Stimuli (G) and response options $(H)$ in a task used in [37] to test for age-related differences in mnemonic discrimination of objects between 4 and 6 years old children, and young adults. After viewing a series of objects without a memory instruction (top), participants had to distinguish targets they saw earlier from highly similar lures and novel foils (bottom). Images reproduced, with permission, from [34] (A-F) and [37] (G,H).

balance between pattern completion and pattern separation is characterized by uneven maturation of regions within the hippocampus.

\section{Human Hippocampal Maturation Continues into Adolescence}

For a relatively long time in the history of cognitive neuroscience, the hippocampus had not been the focus of research on memory development, particularly not in middle childhood until adolescence (Box 1). Investigators were influenced by findings demonstrating that the most robust changes in hippocampal structure occur during the first 2 years of life [40], and that the hippocampus as a whole appeared to be unchanged between the ages of 4 and 25 when assessed longitudinally [41]. Hence, the protracted developmental profile of episodic memory was attributed to neural maturation in brain regions outside the hippocampus or to 
development of hippocampus-cortex interactions. Thus, initial work suggested that memory development during middle childhood and adolescence is mainly driven by maturation of the prefrontal cortex (reviewed in [1]), supported by converging structural [19,42-44] as well as functional neuroimaging $[45,46]$ evidence. However, this view has been challenged when improvements in MRI allowed developmental trajectories of human hippocampus to be mapped in vivo with increasing precision (a recent review on MRI imaging of hippocampal development is given in [47]). As a result, some studies reported data suggesting age-related changes within the hippocampal circuitry that extend beyond childhood and potentially even into adolescence $[41,48]$.

A potential solution to these controversies originated from animal studies reporting histological evidence for markedly different developmental trajectories of regions within the hippocampus of rhesus macaque monkeys in age ranges roughly matching 2-7 years of human age [49]. Specifically, these studies showed that, compared to other hippocampal regions, the DG and the CA3 regions undergo more protracted maturation that continues even beyond the examined period - potentially into early adulthood. These findings provided clear clues for research on human hippocampal development by suggesting that a similarly heterogeneous pattern of development of hippocampal regions may be present in humans. However, this intuition could only recently be tested, owing to technological advances in MRI that allow the acquisition of high-resolution hippocampal images in vivo [32,50,51].

The first study that tested for age-related differences in the structure of regions within the hippocampus using high-resolution MRI in children aged 8-14 years [52] indeed found differential patterns of age effects on regions within the body of the hippocampus, with laterally specific age-related increases of the DG and CA3 until early adolescence, and increases of the CA1 until late adolescence. Similar findings were observed in two recent high-resolution MRI studies of young adults and children of similar age ranges (6-14 [38], and 6-17 [39] years of age). In the former, we observed age-related increases in the DG and CA3, the CA1 and CA2, as well as the subiculum within the hippocampal body, until early adolescence, with increases in DG and CA3 extending into young adulthood (Figure 2A,B); in the latter, the authors observed increases in DG in the body, but decreases in the subiculum in the hippocampal body, and a decrease in CA1 in the hippocampal head. By contrast, one recent study using high-resolution $\mathrm{MRI}$ on a sample of participants aged 8-25 years [53] reported a decrease in DG and CA3 with age. However, this study estimated hippocampal regions based on only the most anterior part of the hippocampal body. Thus, despite slight differences in results (potentially owing to differential demarcation of hippocampal regions) the three studies above assessing regional development within the full extent of the hippocampal body provide converging evidence for protracted hippocampal development characterized by heterogeneous maturation of regions within the hippocampus. Importantly, the available evidence [38] suggests that hippocampal maturation contributes substantially to memory development.

\section{Hippocampal Maturation Shapes the Ontogeny of Memory}

Most crucially, the pattern of age differences in [38] suggests that the uneven maturational trajectories of regions within the hippocampus are related to a shift from pattern completion to pattern separation (Figure 2C). In that study we used multivariate analysis techniques to express the interrelated pattern of age-related differences in all measured regions within the hippocampus, in line with the highly interconnected nature of the hippocampal circuit [54]. Our results are consistent with the hypothesis that the heterogeneous maturation of hippocampal subfields facilitates a shift from generalization to specificity during childhood, and potentially in adolescence. We posit that the lag between generalization and specificity during 


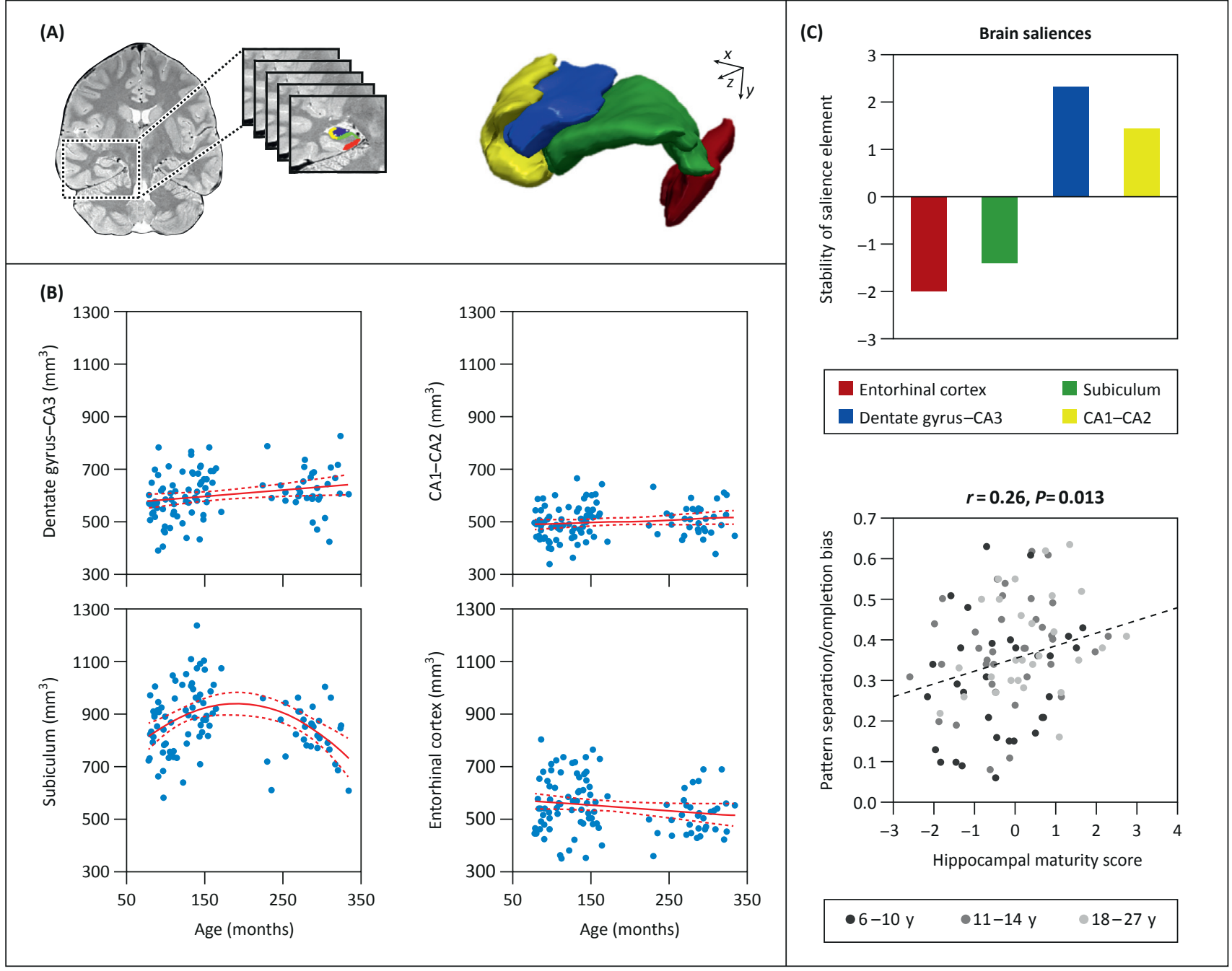

Trends in Cognitive Sciences

Figure 2. Hippocampal Regional Maturation Related to Memory Development. (A) Regions within the body of the hippocampus manually delineated on coronal MRI slices. (B) Hippocampal regions show a heterogeneous pattern of maturation from 6 to 27 years of age that extends well beyond middle childhood [38]. (C, Top) A multivariate vector score (Brain saliences) reflecting the multivariate pattern of age-related differences in hippocampal regions, shows age-related increases in dentate gyrus (DG) and CA3, as well as CA1 and CA2, and age-related decreases in entorhinal cortex and subiculum. The stability of the salience elements suggests that changes in DG and CA3 as well as entorhinal cortex contribute most to the multivariate pattern of hippocampal maturation. (C, Bottom) Hippocampal maturity, capturing the expression of $(\mathrm{C})$ for each individual, is associated with a bias score pitting pattern separation against pattern completion, as measured in a mnemonic similarity task $[32,33]$ similar to the one described in Figure $1 \mathrm{G}, \mathrm{H}$. This suggests that a shift from pattern completion to pattern separation is partly driven by differential maturation of regions within the hippocampus. Images reproduced, with permission, from [38]. Abbreviation: y, years of age.

this life period is developmentally advantageous because it facilitates initial learning through the extraction of commonalities across experiences [1]. According to this view, a shift to more specificity becomes affordable and adaptive only after accumulation of sufficient world knowledge. Of course, we do not claim that the hippocampus is the only player in this developmental shift. In addition to maturational changes within the hippocampus regionally, maturational changes across the entire brain and their connections via white matter pathways [55] are probably driving the ontogeny of learning and memory (Box 1). Future longitudinal studies are faced with the 
difficult and rewarding task of delineating the joint contributions of distributed and uneven maturational brain changes to memory development, and to individual differences therein.

\section{Concluding Remarks and Future Directions}

In this Opinion we have proposed that maturational changes within the hippocampus continue to contribute to the ontogeny of learning and memory throughout childhood and potentially into adolescence. We suggest that this proposition helps to explain the longstanding observation that children tend to extract scripted generalizations of their life experiences, and recollect relatively few details of specific episodes.

To test this proposition, we advocate a neurodevelopmental research program that investigates how interactions among hippocampal regions, in concert with hippocampal-cortical interactions, shape the ontogeny of learning and memory. More specifically, we advocate studying memory using a component process approach [13] informed by (i) computational models of memory based on emerging evidence on the computational properties of memory-relevant neural substrates, and (ii) changes in computational properties as a function of changes in these substrates.

The notion that differential maturational trajectories of regions within the hippocampus drive the ontogeny of generalization and specificity across childhood and adolescence can be further addressed by several lines of research. It has become clear in recent years that hippocampal maturation continues beyond the age of 6 years $[38,47,52,56]$. At the same time, it seems evident that maturational changes in hippocampal circuitry tend to be larger before rather than after that age. To model the developmental trajectory of generalization and specificity under age 6 , and preferably under age 4, the field needs to design novel behavioral assays to probe pattern separation and completion. Similarly, to characterize the maturation of hippocampal regions below 6 years of age, currently available high-resolution MRI protocols need to be adapted for use in younger children ([57] reports a recent success in this direction). The use of faster sequences, online head movement control and correction [58], as well as behavioral interventions [59] will figure prominently in this effort (see also [60]). An intriguing question to be tested by such future studies is whether generalization and specificity may rely on different neural substrates at different stages of hippocampal development. For instance, in light of the relatively immature DG and CA3 in infancy (below 1-2 years), Gómez and Edgin [61] suggested that generalization in infancy occurs mostly incrementally through the cortex. A different computational model by Schapiro et al. [12] suggests that infants can rely also on CA1 to perform fast generalization. Given that CA1 develops relatively early compared to DG and CA3, the fact that there are improvements in tasks tapping into fast generalization well beyond middle childhood [39] may indeed suggest that generalization relies on different mechanisms in infancy and in later stages of childhood development.

In particular, the field needs to increase its efforts to develop tasks that index pattern separation and completion in children. The mnemonic similarity task [33] and its variants typically require mnemonic discrimination between perceptually similar object exemplars. Path-specific pattern separation signals for objects versus scenes within the medial temporal lobe (MTL) have been dissociated in young adults [62], and are differently affected by normal aging in older adults [63]. Hence, structural and functional analyses also need to consider extrahippocampal regions within the MTL - such as the perirhinal cortex and parahippocampal cortex for objects and scenes stimuli, respectively [64].

Finally, neuroimaging studies will profit from successful attempts at more fine-grained delineation of regions within the hippocampus, which has become possible with relatively widespread

\section{Outstanding Questions}

How does hippocampal maturation interact with the development of other cortical regions, and in particular with maturation of the prefrontal cortex?

How can these observations be accommodated by data suggesting a shift from pattern completion to pattern separation during development?

How does the bias towards pattern completion in childhood map onto the processing stages of encoding, consolidation, and retrieval? For instance, are high-resolution details already 'lost' at encoding; that is, is the actual experience immediately converted into a generalizedrepresentation? Orare specifics removed during consolidation(e.g., during sleep)? Do the relative contributions of the three stages to the pattern completion bias change with age?

What is the pattern of hippocampal regional maturation below the age of 6 years?

Can pattern separation and pattern completion be more effectively dissociated at the behavioral level?

How does emotional saliency of experienced episodes interact with tendencies towards generalization versus specificity at different ages?

Does pattern separation operate similarly on different representational contents, including singular items such as individual objects, scenes, and multifaceted contexts such as complex events that comprise disparate elements? If so, how may this interact with differential age effects on item and context memory in children?

What are the boundary conditions that determinethe engagement of hippocampal pattern separation, and how do the boundaries change in development?

Can the link between intrahippocampal maturation and the developmental lag between generalization and specificity inform research on other aspects of child cognitive development, such as the development of prefrontally driven interference resolution during representational conflict, statistical learning, or fear conditioning? 
3 Tesla MRI scanners. These efforts will gain further traction through the establishment of protocols to separate hippocampal regions with greater reliability (e.g., [65]) and validity (e.g., [66]), with a specific focus on separating the DG from the CA3 region [67]. More fine-grained imaging of the hippocampus will also be necessary to extend current findings to the head and tail of the hippocampus. This is essential to fully understand the maturational heterogeneity of regions along the longitudinal axis of the hippocampus, both in terms of structure and function. There is initial evidence for developmental differences along the anterior-posterior axis $[39,41,68]$, but the underlying mechanisms, the significance of these differences, and their links to specific behavioral outputs are not well understood [47].

On a conceptual note, the present article exemplifies the beneficial and reciprocal relations between developmental and general approaches to the neural architecture of cognition. On the one hand, general computational and animal models point to potential reasons for age differences in behavior. On the other, developmental phenomena, such as the lead-lag relation between pattern completion and pattern separation, also help to clarify the role of functional subdivisions within the hippocampal formation.

To conclude, the main proposition of this article is that maturational differences between subfields within the hippocampus contribute to the developmental lead-lag relation between generalization and specificity. The extraction of invariance across a range of different experiences may precede the encoding, consolidation, and retrieval of detail for reasons that are rooted, at least in part, in the uneven maturational course of substructures within the hippocampus. This décalage $[27,28]$, or developmental lag, may be developmentally advantageous because it helps children to recognize regularities, form stable representations of recurring episodes, predict the structure of future events, and build semantic knowledge.

To be firmly established, our main proposition must be put to further empirical test. Crucially, given that change-change relations cannot be inferred on the basis of cross-sectional age differences [69], both the evidence suggesting heterogeneous maturational trajectories within the human hippocampus across childhood and adolescence [38,52], as well as the behavioral evidence for a shift from pattern completion to pattern separation $[34,37,38]$ need to be corroborated by multivariate longitudinal assessments. For instance, a recent study assessing longitudinal change in hippocampal regions between 4 and 22 years of age [70] confirmed the heterogeneous pattern of age-related differences that was previously reported in cross-sectional studies $[38,39,52]$, although with diverging trends for some regions. Future studies need to establish whether these differences reflect methodological discrepancies in delineating hippocampal regions, or perhaps unveil the weakness of inferring development based on cross-sectional data [71]. In designing such future studies, we recommend reliance on (i) experimental materials and procedures that minimize age-associated differences in task-relevant knowledge [37,72], (ii) samples that are sufficiently large to reliably capture change-change relations of moderate effect size, and (iii) a sufficiently large number of measurement occasions to capture the hypothesized lead-lag relations at behavioral and neural levels of analysis.

\section{Acknowledgments}

This work was supported by the German Research Foundation (DFG, WE 4269/5-1 to M.W.B.), the Jacobs Foundation (Early Career Research Fellowship 2017-2019 to M.W.B.), the Max Planck Society, the National Institutes of Health (F31HD090872 to C.T.N.), and the National Science Foundation (SBE-1041707 to N.S.N.). We would like to thank Yee Lee Shing for discussions that contributed to the ideas presented in this Opinion. 


\section{References}

1. Newcombe, N.S. et al. (2007) Development of episodic and autobiographical memory: a cognitive neuroscience perspective. Adv. Child Dev. Behav. 35, 37-85

2. Olson, I.R. and Newcombe, N.S. (2014) Binding together the elements of episodes: relational memory and the developmental trajectory of the hippocampus. In The Wiley Handbook on the Development of Children's Memory (Bauer, P.J. and Fivush, R., eds), pp. 285-308, Wiley-Blackwell

3. Bauer, P.J. (2015) A complementary processes account of the development of childhood amnesia and a personal past. Psychol. Rev. 122, 204

4. Jabès, A. and Nelson, C.A. (2015) 20 years after 'The ontogeny of human memory: a cognitive neuroscience perspective,' where are we? Int. J. Behav. Dev. 39, 293-303

5. Moscovitch, M. (1994) Memory and working with memory: evaluation of a component process model and comparisons with other models. Mem. Syst. 1994, 224

6. Craik, F.I. et al. (1983) On the transfer of information from temporary to permanent memory. Philos. Trans. R. Soc. Lond. B. Biol. Sci. 302, 341-359

7. Tulving, E. and Markowitsch, H.J. (1998) Episodic and declarative memory: role of the hippocampus. Hippocampus 8, 198-204

8. Squire, L.R. and Zola, S.M. (1998) Episodic memory, semantic memory, and amnesia. Hippocampus 8, 205-211

9. Renoult, L. et al. (2012) Personal semantics: at the crossroads of semantic and episodic memory. Trends Cogn. Sci. 16, 550-558

10. McClelland, J.L. et al. (1995) Why there are complementary learning systems in the hippocampus and neocortex: insights from the successes and failures of connectionist models of learning and memory. Psychol. Rev. 102, 419-457

11. Kumaran, D. and McClelland, J.L. (2012) Generalization through the recurrent interaction of episodic memories: a model of the hippocampal system. Psychol. Rev. 119, 573-616

12. Schapiro, A.C. et al. (2017) Complementary learning systems within the hippocampus: a neural network modelling approach to reconciling episodic memory with statistical learning. Philos. Trans. R. Soc. B 372, 20160049

13. Moscovitch, M. et al. (2016) Episodic memory and beyond: the hippocampus and neocortex in transformation. Annu. Rev. Psychol. 67, 105-134

14. Schapiro, A.C. et al. (2014) The necessity of the medial temporal lobe for statistical learning. J. Cogn. Neurosci. 26, 1736-1747

15. Shohamy, D. and Turk-Browne, N.B. (2013) Mechanisms for widespread hippocampal involvement in cognition. J. Exp. Psychol. Gen. 142, 1159-1170

16. Zeithamova, D. and Preston, A.R. (2010) Flexible memories: differential roles for medial temporal lobe and prefrontal cortex in cross-episode binding. J. Neurosci. 30, 14676-14684

17. Gogtay, N. et al. (2004) Dynamic mapping of human cortical development during childhood through early adulthood. Proc. Natl. Acad. Sci. U. S. A. 101, 8174-8179

18. Shing, Y.L. et al. (2008) Associative and strategic components of episodic memory: a life-span dissociation. J. Exp. Psychol. Gen. 137, 495-513

19. Sowell, E.R. et al. (2003) Mapping cortical change across the human life span. Nat. Neurosci. 6, 309-315

20. Kumaran, D. et al. (2016) What learning systems do intelligent agents need? Complementary learning systems theory updated. Trends Cogn. Sci. 20, 512-534

21. Shohamy, D. and Wagner, A.D. (2008) Integrating memories in the human brain: hippocampal-midbrain encoding of overlapping events. Neuron 60, 378-389

22. Schlichting, M.L. and Preston, A.R. (2017) The hippocampus and memory integration: building knowledge to navigate future decisions. In The Hippocampus from Cells to Systems (Hannula, D.E. and Duff, M.C., eds), pp. 405-437, Springer

23. Marr, D. (1971) Simple memory: a theory for archicortex. Philos. Trans. R. Soc. Lond. B Biol. Sci. 262, 23-81
24. Hunsaker, M.R. and Kesner, R.P. (2013) The operation of pattern separation and pattern completion processes associated with different attributes or domains of memory. Neurosci. Biobehav. Rev. 37, 36-58

25. Yassa, M.A. and Stark, C.E. (2011) Pattern separation in the hippocampus. Trends Neurosci. 34, 515-525

26. Rolls, E.T. (2016) Pattern separation, completion, and categori sation in the hippocampus and neocortex. Neurobiol. Learn. Mem. 129, 4-28

27. Chapman, M. (1988) Constructive Evolution: Origins and Deve/ opment of Piaget's Thought, Cambridge University Press

28. Piaget, J. et al. (1977) Epistemology and Psychology of Functions, Reidel

29. Oehrn, C.R. et al. (2015) Human hippocampal dynamics during response conflict. Curr. Biol. 25, 2307-2313

30. Leutgeb, J.K. et al. (2007) Pattern separation in the dentate gyrus and CA3 of the hippocampus. Science 315, 961-966

31. Gillbert, P.E. and Kesner, R.P. (2006) The role of the dorsal CA3 hippocampal subregion in spatial working memory and pattern separation. Behav. Brain Res. 169, 142-149

32. Bakker, A. et al. (2008) Pattern separation in the human hippocampal CA3 and dentate gyrus. Science 319, 1640-1642

33. Stark, S.M. et al. (2013) A task to assess behavioral pattern separation (BPS) in humans: data from healthy aging and mild cognitive impairment. Neuropsychologia 51, 2442-2449

34. Lambert, F.R. et al. (2015) Improvement of allocentric spatia memory resolution in children from 2 to 4 years of age. Int. $J$. Behav. Dev. 39, 318-331

35. Das, T. et al. (2014) Loss of pattern separation performance in schizophrenia suggests dentate gyrus dysfunction. Schizophr. Res. 159, 193-197

36. Yassa, M.A. et al. (2011) Pattern separation deficits associated with increased hippocampal CA3 and dentate gyrus activity in nondemented older adults. Hippocampus 21, 968-979

37. Ngo, C.T. et al. (2018) The ontogeny of relational memory and pattern separation. Dev. Sci. 21Published online March 2, 2017. http://dx.doi.org/10.1111/desc.12556

38. Keresztes, A. et al. (2017) Hippocampal maturity promotes memory distinctiveness in childhood and adolescence. Proc. Natl. Acad. Sci. 114, 9212-9217

39. Schlichting, M.L. et al. (2017) Hippocampal structure predicts statistical learning and associative inference abilities during development. J. Cogn. Neurosci. 29, 37-51

40. Utsunomiya, H. et al. (1999) Development of the temporal lobe in infants and children: analysis by MR-based volumetry. Am. Neuroradiol. 20, 717-723

41. Gogtay, N. et al. (2006) Dynamic mapping of normal human hippocampal development. Hippocampus 16, 664-672

42. Paus, T. et al. (1999) Structural maturation of neural pathways in children and adolescents: in vivo study. Science 283, 1908-1911

43. Giedd, J.N. (2004) Structural magnetic resonance imaging of the adolescent brain. Ann. N. Y. Acad. Sci. 1021, 77-85

44. Sowell, E.R. et al. (2004) Longitudinal mapping of cortical thickness and brain growth in normal children. J. Neurosci. 24, 8223-8231

45. Ofen, N. et al. (2007) Development of the declarative memory system in the human brain. Nat. Neurosci. 10, 1198-1205

46. Cycowicz, Y.M. et al. (2003) Pictures and their colors: what do children remember? J. Cogn. Neurosci. 15, 759-768

47. Lee, J.K. et al. (2017) Hippocampal development: structure, func tion and implications. In The Hippocampus from Cells to Systems (Hannula, D.E. and Duff, M.C., eds), pp. 141-166, Springer

48. Ghetti, S. et al. (2010) Developmental differences in medial temporal lobe function during memory encoding. J. Neurosci. 30, 9548-9556

49. Lavenex, P. and Lavenex, P. (2013) Building hippocampal circuits to learn and remember: insights into the development of human memory. Behav. Brain Res. 254, 8-21 
50. Eldridge, L.L. et al. (2005) A dissociation of encoding and retrieval processes in the human hippocampus. J. Neurosci. 25, 3280-3286

51. Carr, V.A. et al. (2010) Imaging the human medial temporal lobe with high-resolution fMRI. Neuron 65, 298-308

52. Lee, J.K. et al. (2014) Volume of hippocampal subfields and episodic memory in childhood and adolescence. Neuroimage $94,162-171$

53. Daugherty, A.M. et al. (2017) Hippocampal CA3-dentate gyrus volume uniquely linked to improvement in associative memory from childhood to adulthood. Neuroimage 153, 75-85

54. Amaral, D. and Lavenex, P. et al. (2007) Hippocampal neuroanatomy. In The Hippocampus Book (Andersen, P., ed.), pp. 37-114, Oxford University Press

55. Ngo, C.T. et al. (2017) White matter structural connectivity and episodic memory in early childhood. Dev. Cogn. Neurosci. 28, $41-53$

56. Ofen, N. and Shing, Y.L. (2013) From perception to memory: changes in memory systems across the lifespan. Neurosci. Biobehav. Rev. 37, 2258-2267

57. Riggins, T. et al. (2018) Protracted hippocampal development is associated with age-related improvements in memory during early childhood. Neuroimage 174, 127-137

58. Todd, N. et al. (2015) Prospective motion correction of 3D echoplanar imaging data for functional MRI using optical tracking. Neuroimage 113, 1-12

59. Greene, D.J. et al. (2018) Behavioral interventions for reducing head motion during MRI scans in children. Neuroimage 171, 234245

60. Ellis, C.T. and Turk-Browne, N.B. (2018) Infant fMRI: a model system for cognitive neuroscience. Trends Cogn. Sci. 22, 375387

61. Gómez, R.L. and Edgin, J.O. (2016) The extended trajectory of hippocampal development: implications for early memory development and disorder. Dev. Cogn. Neurosci. 18, 57-69

62. Reagh, Z.M. and Yassa, M.A. (2014) Object and spatial mnemonic interference differentially engage lateral and medial entorhinal cortex in humans. Proc. Natl. Acad. Sci. U. S. A. 111, 4264-4273

63. Berron, D. et al. (2018) Age-related functional changes in domainspecific medial temporal lobe pathways. Neurobiol. Aging 65, 86-97

64. Newsome, R.N. et al. (2012) Reducing perceptual interference improves visual discrimination in mild cognitive impairment: implications for a model of perirhinal cortex function. Hippocampus 22, 1990-1999

65. Yushkevich, P.A. et al. (2015) Automated volumetry and regional thickness analysis of hippocampal subfields and medial temporal cortical structures in mild cognitive impairment. Hum. Brain Mapp. 36, 258-287

66. Bender, A.R. et al. (2018) Optimization and validation of automated hippocampal subfield segmentation across the lifespan. Hum. Brain Mapp. 39, 916-931

67. Wisse, L.E. et al. (2017) A harmonized segmentation protocol for hippocampal and parahippocampal subregions: why do we need one and what are the key goals? Hippocampus 27, 3-11

68. DeMaster, D. et al. (2014) Structural development of the hippocampus and episodic memory: developmental differences along the anterior/posterior axis. Cereb. Cortex 24, 3036-3045

69. Raz, N. and Lindenberger, U. (2011) Only time will tell: crosssectional studies offer no solution to the age-brain-cognition triangle: comment on Salthouse (2011). Psychol. Bull. 137, 790-795

70. Tamnes, C.K. et al. (2018) Longitudinal development of hippocampal subregions from childhood to adulthood. Dev. Cogn. Neurosci. 30, 212-222

71. Lindenberger, U. et al. (2011) Cross-sectional age variance extraction: what's change got to do with it? Psychol. Aging $26,34-47$

72. Brod, G. et al. (2017) Neural activation patterns during retrieval of schema-related memories: differences and commonalities between children and adults. Dev. Sci. 20, e12475
73. Tulving, E. (1983) Elements of Episodic Memory, Clarendon

74. Squire, L.R. (1982) The neuropsychology of human memory. Annu. Rev. Neurosci. 5, 241-273

75. Vargha-Khadem, F. et al. (1997) Differential effects of early hippocampal pathology on episodic and semantic memory. Science 277, 376-380

76. Manns, J.R. et al. (2003) Semantic memory and the human hippocampus. Neuron 38, 127-133

77. Wais, P.E. et al. (2006) The hippocampus supports both the recollection and the familiarity components of recognition memory. Neuron 49, 459-466

78. Ranganath, C. and Blumenfeld, R.S. (2005) Doubts about double dissociations between short- and long-term memory. Trends Cogn. Sci. 9, 374-380

79. Axmacher, N. et al. (2007) Sustained neural activity patterns during working memory in the human medial temporal lobe. J. Neurosci. 27, 7807-7816

80. de Haan, M. et al. (2006) Human memory development and its dysfunction after early hippocampal injury. Trends Neurosci. 29, 374-38

81. Cabeza, R. and Nyberg, L. (2000) Imaging cognition II: an empirical review of 275 PET and fMRI studies. J. Cogn. Neurosci. 12, 147

82. Frotscher, M. and Seress, L. et al. (2007) Morphological development of the hippocampus. In The Hippocampus Book (Andersen, P., ed.), pp. 115-131, Oxford University Press

83. Brod, G. et al. (2013) The influence of prior knowledge on memory: a developmental cognitive neuroscience perspective. Front. Behav. Neurosci. 7, 139

84. Anooshian, L.J. (1999) Understanding age differences in memory: disentangling conscious and unconscious processes. Int. J. Behav. Dev. 23, 1-17

85. Brainerd, C.J. et al. (2004) Behavioral measurement of remembering phenomenologies: so simple a child can do it. Child Dev. 75, 505-522

86. Ghetti, S. and Angelini, L. (2008) The development of recollection and familiarity in childhood and adolescence: evidence from the dual-process signal detection model. Child Dev. 79, 339-358

87. McHugh, T.J. et al. (2007) Dentate gyrus NMDA receptors mediate rapid pattern separation in the hippocampal network. Science 317, 94-99

88. Nakashiba, T. et al. (2012) Young dentate granule cells mediate pattern separation, whereas old granule cells facilitate pattern completion. Cell 149, 188-201

89. Sahay, A. et al. (2011) Pattern separation: a common function fo new neurons in hippocampus and olfactory bulb. Neuron 70 , 582-588

90. Leutgeb, S. and Leutgeb, J.K. (2007) Pattern separation, pattern completion, and new neuronal codes within a continuous CA3 map. Learn. Mem. 14, 745-757

91. Rolls, E. (2013) The mechanisms for pattern completion and pattern separation in the hippocampus. Front. Syst. Neurosci. 7,74

92. Horner, A.J. and Burgess, N. (2014) Pattern completion in multielement event engrams. Curr. Biol. 24, 988-992

93. Horner, A.J. et al. (2015) Evidence for holistic episodic recollection via hippocampal pattern completion. Nat. Commun. 6 , ncomms8462

94. Danker, J.F. et al. (2017) Trial-by-trial hippocampal encoding activation predicts the fidelity of cortical reinstatement during subsequent retrieval. Cereb. Cortex 27, 3515-3524

95. Santoro, A. (2013) Reassessing pattern separation in the dentate gyrus. Front. Behav. Neurosci. 7, 96

96. Bartlett, F.C. (1932) Remembering, Cambridge University Press

97.. Kirwan, C.B. and Stark, C.E.L. (2007) Overcoming interference: an fMRI investigation of pattern separation in the medial temporal lobe. Learn. Mem. 14, 625-633 